\title{
SOAP Revisitado
}

Maria João Queiroz*

\section{RESUMO}

O registo clínico é uma ferramenta essencial na gestão clínica. É um suporte informativo e meio de comunicação, reflecte a qualidade dos cuidados médicos, contribui para a formação e para a investigação. Em Medicina Geral e Familiar a necessidade de organizar os dados provenientes de cuidados prestados de forma contínua e por longos períodos vem relançar o tema, reanalisar a pertinência da utilização da metodologia habitualmente seguida, nomeadamente avaliar a actualidade do SOAP e a sua adaptabilidade face às novas aplicações no registo.

A propósito do método, este artigo salienta as suas virtuosidades e os seus pontos fracos, e sugere algumas estratégias para lhes fazer face.

Palavras-Chave: Registo clínico; SOAP.

\section{INTRODUÇÃO}

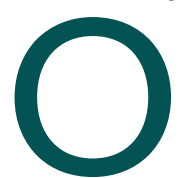
registo clínico tem assumido um interesse crescente em Medicina Geral e Familiar (MGF), dependente da necessidade de melhorar a eficiência dos serviços assistenciais. Este maior protagonismo converteu o bom registo clínico numa das principais ferramentas do médico de família, ${ }^{1}$ nomeadamente para a gestão clínica, encarada tanto do ponto de vista do doente individualmente considerado, como do ponto de vista da gestão e avaliação dos serviços. ${ }^{2} \mathrm{O}$ registo clínico é um instrumento operacional e um componente decisivo dos cuidados médicos, que contribui para a sua qualidade e a reflecte. No projecto MoniQuOr os registos clínicos foram alvo de avaliação por serem essenciais à continuidade de cuidados, para o desenvolvimento científico e para a protecção médico-legal. ${ }^{3}$

O médico de família presta cuidados por longos períodos de tempo aos mesmos utentes. A exemplo, em Portugal, grande parte dos médicos de família mantém a mesma lista de utentes há cerca de duas décadas. Este facto obriga a coleccionar e gerir um manancial muito volumoso de dados informativos. A tarefa de registar deve ser encarada como uma necessidade de traduzir no presente, de forma fiel, o estado de saúde do doente, assim como de, no futuro, planear e monitorizar o seu acompanhamento. É obrigação do registo ordenar a informação colhida, facilitando a reflexão para a to-

* Médica de Família, Chefe de Serviço no CS de Venda Nova Assistente Convidada da Cadeira de Medicina Geral e Familiar, da Faculdade de Ciências Médicas da Universidade Nova de Lisboa mada de decisões, ${ }^{4}$ para a auto-aprendizagem e para a comunicação interpares.

Segundo Weed $^{5}$ o registo não é um repositório estático de observações médicas estruturadas, mas antes um instrumento de comunicação e só um registo organizado pode ser considerado um documento científico. Assim nasceu, por si proposto, o Registo Médico Orientado por Problemas (RMOP), de que a MGF se apropriou desde a década de 70 , trazendo-o até à actualidade e adaptando-o inclusivamente a suportes informáticos, apesar de ter sido criado para o registo em papel, facto que bendiz da sua versatilidade.

É objectivo deste artigo fazer uma viagem ao longo da vida útil deste método, salientar as suas virtuosidades nomeadamente da sua componente SOAP, assim como chamar a atenção para as limitações que lhe são inerentes, apontando alguns caminhos para as contornar.

\section{OBJECTIVOS}

$O$ registo é sobretudo um suporte informativo, reflectindo o estado de saúde do utente, e um meio de comunicação, permitindo comunicar factos relevantes. Contribui para as cinco áreas no âmbito da actividade médica:

1) Auxiliar de memória

2) Transferência de informação

3) Avaliação de qualidade

4) Formação

5) Investigação

Em MGF a consulta faz parte de uma série, tem efei- 
tos cumulativos e um contexto cronológico e está integrada num ciclo de cuidados. Esta complexidade relativa a cada utente, multiplicada por muitos, obriga o médico a socorrer-se de uma memória escrita, extensível, dinâmica e eficaz. Os dados armazenados servem o médico assistente ou outro que transitória ou definitivamente o substitua, na medida em que o processo clínico acompanha a transferência do doente, no Serviço Nacional de Saúde. É também do processo clínico que são transferidos dados para outras bases de dados (Registo Oncológico Regional, DiabCare, entre outros) ou elaborados relatórios para referenciação, para outras finalidades ou a pedido do doente.

O registo clínico é por vezes a única fonte que permite recolher informação sobre a actividade clínica pelo que fornece dados para auditorias e investigação judicial. Na base da auditoria pode estar a necessidade de comparar a performance do indivíduo face ao grupo, avaliando a qualidade técnico-científica ou outra das dimensões da qualidade, corrigir vícios de atitude, mas serve sempre objectivos didácticos. A avaliação dos registos deve verificar o «conteúdo clínico essencial, sem excessos e facilmente colectável e localizável». ${ }^{6}$ Não basta fazer bons registos para se fazer boa medicina, ${ }^{7}$ mas há uma correlação entre a qualidade do registo e a qualidade da prática. Também em tribunal, o registo actualizado e completo é a melhor defesa: para a justiça um acontecimento não registado é um acontecimento inexistente. Segundo o Código Deontológico da Ordem dos Médicos (art. 77º), «o médico...tem o direito e o dever de registar cuidadosamente» e pode incorrer em pena disciplinar por falta nos actos de organização e funcionamento. ${ }^{8}$

O adulto aprende através da síntese do conjunto de influências exteriores, tendo em conta os seus contextos, e apropria-se do seu próprio processo de formação. ${ }^{9}$ A confrontação sistemática do actor (médico) com as suas acções, que na prática médica é decorrente da verificação incontornável no doente, do impacto das medidas tomadas, vai facilitar a auto-análise. O registo dá uma preciosa ajuda: sinaliza erros e omissões, reforça conhecimentos, aptidões e atitudes que estão na base dos procedimentos clínicos. O treino do registo clínico pode ser uma estratégia de ensino-aprendizagem porque de forma indirecta se reflecte sobre a consulta. ${ }^{10}$

A revisão de ficheiro, para além de poder identificar lacunas formativas, pode fazer sobressair problemas que mereçam ser investigados. É também ao ficheiro clínico que se pede frequentemente contributo numa fase inicial dos estudos descritivos para investigação clínica.

McWhinney ${ }^{11}$ identifica também cinco objectivos mais operativos, dado que a informação deve estar dirigida para a acção. Segundo Zurro, gerir implica escolher entre alternativas diferentes, implica emitir um juízo baseado na análise rigorosa da informação adequada. São nomeados da seguinte forma:

1) Aceder rapidamente aos dados básicos do doente

2) Fornecer dados contínuos sobre os problemas de saúde

3) Acompanhar as consultas do doente

4) Fornecer dados para organizar medidas preventivas

5) Contribuir para a educação contínua

A boa acessibilidade da informação e a facilidade na sua circulação melhoram a performance da equipa de saúde, potenciam a relação do utente com os seus diferentes elementos e multiplicam oportunidades de educação para a saúde.

O registo contínuo beneficia sobretudo os doentes com doença crónica, devendo reflectir em qualquer momento o grau de controlo da doença e os problemas passados que possam fazer prever problemas potencialmente significativos decorrentes do estado de saúde actual (ex. de doente diabético não compensado, com internamento no passado por coma diabético, ou o caso do doente hipocoagulado, com INR instável).

O registo sistemático das consultas do doente permite caracterizar o perfil de consumo de cuidados médicos e a sua cronologia, informações que podem orientar o médico para a verdadeira natureza subjacente aos problemas apresentados. Os indícios podem ser corroborados se outros elementos do agregado familiar reincidirem no mesmo padrão de consumo.

O facto dos registos traduzirem os cuidados de saúde prestados por longos períodos de tempo ao doente melhoram a planificação de actividades preventivas. $\mathrm{O}$ acesso ao registo de datas de procedimentos preventivos prévios permite calendarizar os próximos.

Para além do que foi dito sobre formação contínua, a continuidade de cuidados e consequentemente de registos dão a oportunidade ao médico de conhecer a história natural das doenças. 


\section{RMOP - SOAP}

O RMOP é um sistema de resolução de problemas. Fez o ano passado 40 anos e a MGF portuguesa praticamente desde o seu início, há cerca de 30 anos, o adoptou e implementou como método de registo. Continua a responder às necessidades da actualidade e, apesar da importância e especificidades de cada uma das suas componentes, é sobre as notas de seguimento ou progressivas SOAP e a gestão do seu conteúdo que se fazem as considerações a seguir.

- «S» de Subjectivo regista o ponto de vista do doente - para além das queixas, os sentimentos.

- «O» de Objectivo traduz o ponto de vista do médico, com dados registados sem viés, incluindo os resultados de testes de diagnóstico.

- «A» de Avaliação identifica o problema e o seu grau de resolução à data. A formulação dos problemas deve ser o mais precisa possível, evitando-se conjecturas com base em dados para além dos disponíveis: pode ser um sintoma ou um sinal. O problema deve constituir um «facto clínico», ${ }^{12}$ excluindo dúvidas, interrogações ou negações, porque uma hipótese reduz a amplitude da investigação clínica. A hipótese diz respeito ao médico que a coloca e não ao doente. É o «A» que alimenta a lista de problemas, exclusão feita às situações agudas auto-limitadas e que não apresentam relevância para o seguimento futuro do doente.

- «P» de Plano propõe as medidas terapêuticas, os pedidos de exames complementares de diagnóstico (ECD), a referenciação e o aconselhamento feito, e agenda a próxima consulta de reavaliação. O plano deve questionar a importância do problema para o doente, para além das investigações a empreender.

\section{PONTOS FORTES - VIRTUOSIDADES}

A valorização da continuidade de cuidados em MGF implicou a criação de um sistema que respondesse à necessidade da gestão da multiplicidade dos dados. $\mathrm{O}$ SOAP evita uma solução de continuidade na transmissão da informação, tornando-se por excelência no método de registo longitudinal. Há que referir, no entanto, que a independência relativa de cada episódio de consulta registado permite a sua análise transversal, facto oportunamente usado para fins diversos, como é o caso, entre outros da formação contínua, ou da colhei- ta de dados para outras bases de dados.

O SOAP é um sistema racional de registo por duas razões principais: primeiro, porque é a tradução escrita resumida, objectiva e despida de detalhes supérfluos, dum episódio de consulta e, segundo, porque as suas componentes «S», «O», «A» $\mathrm{e}$ «P» se podem correlacionar com os passos da consulta. ${ }^{13}$ Poder-se-á acrescentar uma terceira, que é a harmonia na cascata: Consulta $\rightarrow$ Registo $\rightarrow$ Codificação.

A pedra de toque do SOAP como nota de seguimento reside no facto da sua estrutura manter uma sequência temporal em episódios com identidade e datação próprias, independentes, facilmente identificáveis. A organização dos dados usando estas «4 letras» em posições fixas e sequenciais, em que o mesmo tipo de dados é sempre registado na mesma posição relativa, facilita a legibilidade e a procura retrospectiva de um dado específico, como por exemplo percorrer os «P» para aceder à data do pedido de um determinado exame para diagnóstico ou do envio à consulta hospitalar. Este facto permite poupar tempo, pois alguns estudos referem que metade do tempo de consulta é gasto pelo médico na leitura dos registos, a fazer anotações e a atender o telefone, em detrimento da atenção prestada ao doente. ${ }^{14}$

O Método permite registar muita informação em poucas palavras, evitando dados acessórios que apenas causam «ruído». Registam-se exclusivamente os dados referentes aos problemas que é pertinente tratar na consulta em questão, estando respeitada a cronologia dos acontecimentos. Na consulta seguinte, facilita obter feed-back dos procedimentos efectuados e assim actualizar a lista de problemas e ajustar o plano.

A medicação tem sido considerada a componente mais importante da nota de seguimento, devendo por isso estar bem identificada de forma que tanto o médico assistente como alguém que o substitua lhe acedam facilmente.

A colheita de dados retirada deste método de registo facilita as auditorias, dada a sua grande objectividade e pequena ambiguidade. É fácil ao auditor perceber a lógica da abordagem clínica, facilmente correlaciona o plano com a avaliação, a colheita de dados objectivos com as queixas apresentadas, não havendo necessidade de especular sobre as intenções do médico. 


\section{PONTOS FRACOS - VICISSITUDES}

A multiplicidade de consultas pelo mesmo motivo clínico pode conduzir à recolha repetitiva de elementos pouco informativos que nada acrescentem ao que se quer saber sobre o doente, multiplicando informação excedentária, de interesse transitório, soterrando e dispersando os dados verdadeiramente importantes.

Podem identificar-se alguns aspectos negativos relacionados com o registo em geral e de forma específica com cada um dos patamares do SOAP.

Os efeitos de uma consulta podem influenciar a consulta seguinte por provocarem alterações na saúde do doente assim como podem melhorar a sua compreensão da saúde, o que é determinante para a melhoria do ciclo de cuidados. A clarificação de dúvidas, mitos e crenças permite conhecer o contexto do doente, compreender as suas atitudes, e como consequência ajudar a «lidar» com os seus problemas de saúde. Muitos problemas que o doente traz à consulta são de natureza psicológica ou social, sendo necessário explorar as suas ideias, preocupações e expectativas e a forma como pensa poder ser ajudado pelo médico. Muitos problemas não podem ser tratados, mas têm de ser cuidados, ajudando o doente a lidar com a situação. Prever as reacções emocionais esperadas e registá-las, de forma a pressupor o efeito de qualquer intervenção na doença e na vida do doente, melhora a compreensão sobre as preocupações e comportamentos do doente. Segundo McWhinney, a incapacidade do médico para lidar com este tipo de problemas leva a consultas disfuncionais e à medicalização da vida. Daqui decorrente, advém o comprometimento da qualidade do registo Subjectivo e Objectivo: os dados que se reportam a comportamentos, sentimentos, emoções, preocupações, preconceitos e ideias acerca da natureza dos problemas, estão subnotificados. ${ }^{15}$ A linguagem não verbal é também de difícil tradução escrita. A subnotificação compromete o feedback podendo pôr em causa a consulta seguinte e o ciclo de cuidados.

A Avaliação está também sujeita a subnotificação, nomeadamente dos problemas sociais e dos factores de risco.

O Plano executivo tem pontos fracos que se relacionam com as duas últimas das suas três subdivisões 1) ECD, 2) medidas terapêuticas e 3) medidas educativas. A medicação crónica registada no plano apenas se reporta aos problemas avaliados na consulta, ficando de fora toda a prescrição referente a outras afecções. É por isso difícil manter a medicação actualizada, ocorrendo omissões frequentes, o que não raramente conduz a erros grosseiros. Estas falhas no registo são potenciadas pelas consultas telefónicas ou quando as alterações são propostas a terceiros, frequentemente outro familiar que aproveita a vinda à consulta para também pôr questões e fazer pedidos em nome de outro, ou ainda por modificações introduzidas nas consultas hospitalares .

A educação do doente e as medidas preventivas e de apoio propostas são dados habitualmente negligenciados no registo. O registo sistemático do aconselhamento disciplina o médico para a procura de soluções negociadas, para a partilha de informação e para a atribuição de tarefas que impliquem mais o doente no seu processo terapêutico.

\section{O FUTURO}

De uma forma geral poder-se-á afirmar que há que ultrapassar as duas maiores dificuldades ligadas ao registo em MGF: o excesso de dados e a sua omissão. Em relação ao primeiro, é necessário expurgar dados supérfluos, fazendo resumos periodicamente para sumarizar a informação importante.

Há várias medidas para combater a omissão de dados, dependendo das situações em que ocorre:

1) Melhorar o registo da medicação

A actualização do registo sobre a medicação crónica é fundamental. Na literatura médica há várias propostas para atenuar o problema e a que colhe maior aceitação é o registo em folha própria para prescrição, onde esteja contemplada a posologia, número de doses e horário de tomas, a suspensão e eventual motivo.

2) Melhorar o registo das oportunidades preventivas A actualização da morbilidade permite identificar pacientes em risco e estabelecer um plano preventivo. As actividades preventivas só respeitam o calendário preconizado se houver um sistema que avise o médico da necessidade e do momento de aplicação. Precisa-se assim de construir um registo «memo» que, de forma sistemática, recorde os procedimentos a aplicar a cada doente. Há que pôr em marcha o Mapa de Procedimentos, que nas linhas propõe os 
procedimentos preconizados de acordo com o seu grau de evidência, e nas colunas, as idades-chave em que devem ser efectuados. Os resultados podem ficar anotados nas intersecções, utilizando o código de $\mathrm{N}$ ou A, para normal e anormal, respectivamente.

3) Melhorar o registo do aconselhamento

O registo do aconselhamento é um aspecto sobretudo importante nas doenças crónicas e nos factores de risco ligados aos estilos de vida. São disso exemplo o pedido de auto-controlo glicémico feito ao doente diabético; a folha de aconselhamento alimentar distribuída ao utente com dislipidémia; o aconselhamento feito sobre exercício físico; a proposta de ajuda para deixar de fumar ou a negociação feita sobre a quantificação e datação de perda de peso, no doente obeso.

O doente, para além de ser portador da Folha de Prescrição e de alguns panfletos informativos, deve, sobretudo em caso de urgência ou em viagem, ter um «passaporte de saúde» onde constem os seguintes problemas: enfarte de miocárdio, diabetes mellitus, alergias medicamentosas, medicação hipocoagulante e o estado vacinal.

O registo médico electrónico (RME) pode introduzir melhoria global no registo, inclusivamente diminuir os custos com a saúde. Como refere o recém-eleito presidente Obama, «We will make sure that every doctor's office and hospital in this country is using cutting edge technology and electronic medical records so that we can cut red tape, prevent medical mistakes, and help save billions of dollars each year». ${ }^{16} \mathrm{Em} 2005$ previu-se uma poupança de 81 biliões de dólares devido à implementação do RME. ${ }^{17}$

A legibilidade é a primeira qualidade a ser beneficiada, para além de fazer a gestão eficaz de grande volume de informação disponível. A rapidez de acesso, a capacidade de armazenamento, a segurança com a possibilidade de fazer cópias de segurança, a poupança, a versatibilidade, podendo usar códigos para vários utilizadores, a facilidade de arquivo e a interface com outros programas e registos, a troca de informação, o acompanhamento de processos, a organização de actividades, assim como a análise estatística da informação, são aspectos não menos importantes. Mas muitos outros verbos podem caracterizar os ficheiros electrónicos: catalogar, arquivar, distribuir, consultar, editar, publicar.

Quando se faz o registo informático há que ter em conta a mudança na direcção do olhar, com potencial negligência de atenção ao doente, como refereVictor Ramos. Outros pontos que limitam a qualidade dos ficheiros electrónicos são a sua indisponibilidade por falha do sistema, a morosidade no registo, sobretudo a introdução de alguns dados específicos, a menor evidência do perfil de consumo, estando oculto «o processo gordo».

O eventual comprometimento da relação médico-doente, devido à intrusão do computador como uma «terceira pessoa», pode ser minimizado utilizando estratégias que demonstrem ao doente que o médico está interessado em si, como seja fazer pequenas pausas no registo e procurar com frequência o contacto visual, sobretudo em momentos chaves da consulta, e mantê-lo quando se põem questões mais delicadas e enquanto se ouvem as respectivas respostas. Nas consultas cujo motivo se relacione com sofrimento psicológico evidente, com expressão emocional intensa, o registo poderá mesmo ser diferido para um tempo pós consulta. O computador poderá ser integrado na consulta na presença do doente, através da sua humanização, falando dele ao doente como «o meu aliado», «a minha segunda memória», e também pela demonstração das vantagens, como é exemplo a monitorização facilitada da prescrição, destronada a tradicional «letra de médico», com a consequente diminuição dos erros de leitura conducentes à troca de medicamentos dispensados na farmácia.

A confidencialidade dos dados, devido à sua circulação mais livre, pode ser posta em causa. Assim, o registo «apenas à distância de um clique» pode correr o risco de ser suplantado pelo registo em papel ou, pelo menos, não o substituir definitivamente. Habitualmente, o Registo Médico é considerado um sistema híbrido, compreendendo documentação electrónica e em papel. ${ }^{18}$ Esta duplicidade decorre também da dificuldade da adaptação do RMOP ao desenvolvimento de software adequado. Na década de 90 foi implementado o sistema OHEAP (Orientação, História, Exame, Avaliação e Plano) para ultrapassar as dificuldades ligadas à utilização informática do SOAP. ${ }^{19}$ Enquanto a lista de problemas teve aceitação generalizada, o seu desenho e a sua função em experiências de desenvolvimento foram variadas e inconsistentes, com falta de acordo universal e grande confusão sobre os dados padrão. Foram tidas como necessárias as seguintes qualidades da lis- 
ta de problemas para o sucesso da implementação informática dos registos clínicos: centralidade clínica, codificação dos problemas, apoio para a resolução de problemas, historicidade dos problemas, apoio para diferentes opiniões clínicas, integração das funções do circuito processual, apoio ao registo administrativo, integração de ferramentas clínicas.

O software para o registo clínico disponível nos Centros de Saúde usa o SOAP como método de registo. O seu manejo torna-se moroso e difícil para o utilizador na medida em que os programas informáticos que o suportam (nomeadamente o SAM) vivem mais centrados nas duas funcionalidades inicialmente desenvolvidas a agenda médica e a prescrição. $\mathrm{O}$ facto do doente não ser a centralidade deste processo criou algumas deficiências das quais se destacam o acesso limitado aos dados por abertura sucessiva de campos de forma progressiva e regressiva frequente; o registo dos dados clínicos feito com alguma complexidade, havendo necessidade de digitar os mesmos dados em quadros múltiplos; 0 acesso aos meios complementares de diagnóstico e terapêutica organizados de forma pouco intuitiva (exemplo, entre outros, da prescrição dos tratamentos de fisioterapia); a inacessibilidade dos dados para revisão do ficheiro e do seu tratamento após cinco dias da data da consulta.

É necessário limar as arestas, corrigir os defeitos e melhorar estes suportes simplificando-os, tornando-os mais atractivos a fim de generalizar a sua utilização. Um objectivo mais ambicioso será introduzir as alterações necessárias para melhorar a interacção entre os programas utilizados nas diversas instituições de saúde de forma a que a transferência e tratamento dos dados seja feito com menor esforço possível.
REFERÊNCIAS BIBLIOGRÁFICAS

1. Rakel RE. Textbook of Family Practice. 4th ed. Philadelphia:W. B. Saunders; 1990. p. 1693-795.

2. Zurro AM, Pérez JF. Atención Primaria: conceptos, organización y práctica. Vol. 1. $4^{\mathrm{a}}$ ed. Madrid: Harcourt; 1999. p. 258-74.

3. Direcção-Geral de Saúde. Monitorização da Qualidade Organizacional dos Centros de Saúde - MoniQuOr. $2^{\text {a }}$ ed. Lisboa: DGS; 1999. p. 31.

4. Caeiro R. Registos clínicos em medicina familiar. Lisboa: Instituto de Clínica Geral da Zona Sul; 1991.

5. Weed LL. Medical records that guide and teach. N Engl J Med 1968; 278: 593-600, 652-7.

6. Fragata J, Martins L. O erro em medicina: perspectivas do indivíduo, da organização e da sociedade. Coimbra: Almedina; 2008.

7. Reed DE. The Weed system and complete records. Arch Intern Med 1972 May; 129 (5): 834-5.

8. Pina JA. A responsabilidade dos médicos. $3^{\mathrm{a}}$ ed. Lisboa: Lidel; 2003.

9. Nóvoa A. A formação tem de passar por aqui: as histórias de vida no projecto Prosalus. In: Nóvoa A, Finger M, orgs. Lisboa: Departamento de Recursos Humanos da Saúde; 1988.

10. Broeiro P. Multipatologia: O raciocínio clínico e a tomada de decisão: aquisição de competência. Rev Port Clin Geral 2001 Jul-Ago; 17 (4): 307-26.

11. Mc Whinney IR. Manual de Medicina Familiar. Lisboa: Inforsalus; 1994.

12. Barreto JV, Paiva P. O registo clínico orientado por problemas. Rev Soc Port Med Int 2008 Jul-Set; 15 (3): 201-6.

13. Ramos V. A Consulta em 7 Passos. Lisboa:VFBM Comunicação; 2008.

14. Enelow AJ, Forde DL, Brummel-Smith K. Entrevista clínica e cuidados ao paciente. Lisboa: Climepsi; 1999.

15. Pendleton D, SchofieldT, Tate P, Havelock P. A consulta: uma abordagem à aprendizagem e ensino. Porto: Departamento de Clínica Geral da Faculdade de Medicina do Porto; 1993.

16. Mahar M. Irrational exuberance over electronic medical records. Health Beat 2008 Dez 17. Disponível em: http://www.healthbeat.org [acedido em 30/04/2009].

17. Hillestad R, Bigelow J, Bower A, Girosi F, Meili R, Scoville R, et al. Can electronic medical record systems transform health care? Potential health benefits, savings, and costs. Health Aff (Millwood) 2005 Sep-Oct; 24 (5): 1103-17.

18. Corporate Compliance Policies and Procedures. Legal Medical Record Standards: Policy NO. 9420, 05/01/2008. Disponível em: http:// www.ucop. edu/ucophome/coordrev/policy/legal-medical-record-policy.pdf [acedido em 30/04/2009].

19. NHS - Information Authority. Problem Oriented Medical Record (POMR) and SOAP. 1999; Out. Disponível em: http://www.prorec.it/ documenti/EPR_EHR/NHS-Update-POMR-SOAP.doc [acedido em 30/04/2009].

ENDEREÇO PARA CORRESPONDÊNCIA

Maria João Queiroz

Rua Dr. Mascarenhas de Melo, nº 3 - R/C Esq. 1500-245 Lisboa

\section{ABSTRACT}

The medical record is an essential tool for clinic management. Its contributions are invaluable: as an informational support, as a communication tool, as a medical care evaluator and as a research and traineeship contributor. In General Practice it is critical to create an organized and sustainable data storage for medical care. That need brings to discussion the relevance of using the old fashioned methods, especially SOAP, and its adaptability towards the new record applications.

This article highlights SOAP strengths and flaws, suggesting some strategies to overturn them.

Keywords: Medical Records; SOAP. 